\title{
A State-Space Degradation Model with Multiple Observations and Different Sampling Times
}

\author{
Xianglong $\mathrm{Ni}^{*}$, Xin Zhang, Jianmin Zhao, and Haiping Li \\ Mechanical Engineering College, Shijiazhuang, 050003, China
}

\begin{abstract}
A traditional state-space model (SSM) only contains one observation equation. There are some restricted conditions when using the traditional SSM to describe the evolution process between a state indicator and multiple observation indicators instantaneously. In order to solve this problem, this paper puts forward an SSM that has multiple observation equations, which can be applied to multiple observation indicators with different sampling times. The modeling process and parameters evaluation approach of the proposed SSM are studied and given. A simulation study is conducted to indicate advantages of the proposed SSM when sampling times and observation equations are not the same for different observation indicators. Simulation results show that the proposed SSM is more accurate than the traditional SSM in system degradation prediction.
\end{abstract}

Keywords: state-space model; multiple observation equation; parameter estimation; degradation prediction

(Submitted on May 2, 2016; First revised on June 27, 2017; Second revised on December 3, 2017; Accepted on December 25, 2017)

(O2018 Totem Publisher, Inc. All rights reserved.

\section{Introduction}

Some state indicators can directly reveal system degradation states. However, these indicators are often unable or are difficult to directly measure, such as abrasion, corrosion, crack, etc. Meanwhile, some observation indicators can indirectly reflect system degradation states, and these observation indicators can be obtained easier than state indicators, such as vibration signals, oil data, temperature, and so on. As SSM can establish the relationship between system state indicators and observation indicators [3], and it is usually used to describe a system degradation process through indirect observation indicators.

A state-space model with a single observation equation (SSM-SOE) is the most widely used SSM in existing study. A state indicator often only considers an indirect observation indicator in SSM-SOE. If SSM-SOE is used to describe a state indicator that has multiple observation indicators, there are two conditions that must be met: (a) Sampling times of all observation indicators must be the same and (b) Observation equations must be the same, which describe the relationship between system state indicator and different observation indicators. For example, Orchard used a non-linear SSM to predict axial crack growth of UH-60 planetary gearbox by only analyzing the vibration-based feature [6]. Sun developed SSM for prognostics of gas turbine subject to degradation by monitoring temperature, and there is only one observation equation in SSM [7]. In order to develop the effectiveness of life prediction based on SSM, Zhou used mean square error (MSE) and contribution ratio to choose an appropriate observation indicator from multiple observation indicators, but he only considered an observation indicator in the state estimation process $[8,9]$.

However, as condition monitoring technology develops, there is more than one observation indicator in system condition monitoring in some cases. For example, both vibration data and oil data are collected in the condition monitoring of some mechanical systems (such as engine, gearbox). In bearing condition monitoring, management systems not only monitor bear vibration date, but also bear pulse data by a shock pulse method (SPM) [2]. In these cases, the observation indicators are more

\footnotetext{
* Corresponding author.

E-mail address: ni_phm@163.com
} 
than one in condition monitoring, sampling times of different observation indicators are not the same, and the relationship between system state indicator and different observation indicators are not the same as well. Therefore, SSM-SOE cannot be applicable to these cases.

In order to solve this problem, this paper puts forward a state-space model having multiple observation equations (SSMMOE), which can be applied to multiple observation indicators with different sampling times.

\section{Model formulation}

The SSM-SOE is shown as formula (1), and there is only one observation equation.

$$
\left\{\begin{array}{c}
x_{i+1}=F\left(x_{i}, \vartheta\right)+\xi \\
y_{i}=H\left(x_{i}, \varphi\right)+\varepsilon
\end{array}\right.
$$

In SSM-MOE, which is proposed in this study, the number of observation equations should be established as the number of observation indicators. In other words, when there are $n(n=1,2,3, \ldots)$ observation indicators in system condition monitoring, the established SSM should have $n$ observation equations. The common format of SSM-MOE can be expressed as:

$$
\left\{\begin{array}{c}
x_{i+1}=F\left(x_{i}, \vartheta\right)+\xi \\
y_{i}^{(1)}=H^{(1)}\left(x_{i}, \varphi^{(1)}\right)+\varepsilon^{(1)} \\
y_{i}^{(2)}=H^{(2)}\left(x_{i}, \varphi^{(2)}\right)+\varepsilon^{(2)} \\
\vdots \\
y_{i}^{(n)}=H^{(n)}\left(x_{i}, \varphi^{(n)}\right)+\varepsilon^{(n)}
\end{array}\right.
$$

Where, $x_{i}(i=1,2, \cdots, m)$ is the system degradation state at working time $t_{i}, y_{i}^{(j)}$ is the $j$ th $(j=1,2, \cdots, n)$ observation indicator of $x_{i}, F(\cdot)$ is the degradation state evolution equation, $H^{(j)}(\cdot)$ is the observation equation of $y_{i}^{(j)}, \vartheta$ is the function parameter of $F(\cdot), \varphi^{(j)}$ is the function parameter of $H^{(j)}(\cdot), \xi$ and $\varepsilon^{(j)}$ are noises of $F(\cdot)$ and $H^{(j)}(\cdot)$, respectively.

As shown in Figure 1 ( $x_{0}$ is system degradation initial state), there are some characteristics in SSM-MOE: (a) For degradation state $x_{i}$, observation indicators $y_{i}^{(j)}$ and $y_{i}^{(l)}$ are not needed to exist at the same time, $l, j=1,2, \cdots, n, l \neq j$. (b) Observation equation $H^{(j)}(\cdot)$ and $H^{(l)}(\cdot)$ may be different.

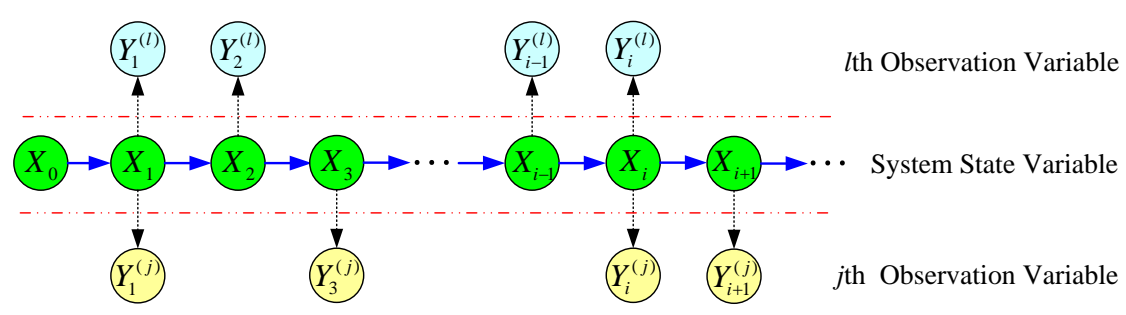

Figure 1. The relationship between system state indicator and multiple observation indicators

\section{Parameter estimation}

Because the sampling times of different observation indicators might be different, it is not fit for selecting observation indicators according to MSE or contribution ratio as Zhou proposed in references [8,9]. Meanwhile, the number of model parameters in SSM-MOE is too high to evaluate, and model parameters evaluation cannot be completed just one-time as SSMSOE. Therefore, model parameters should be separately evaluated.

As shown in Figure 2, in the $p$ th $(p=1,2, \cdots, L)$ iteration of SSM-MOE parameter estimation process, the model parameters estimation steps are given as follows: 
(a) Break up SSM-MOE into $n$ SSM-SOE.

(b) Estimate model parameters of every SSM-SOE. In parameters estimation process of SSM-SOE, $\vartheta_{p}^{(j)}, \xi_{p}^{(j)}, \varphi_{p}^{(j)}, \varepsilon_{p}^{(j)}$ are the $p$ th iteration assessment results according to state indicator $x$ and the $j$ th observation indicator $y^{(j)}$.

(c) Comprehensive analysis state equation parameters $\vartheta_{p}$ and $\xi_{p} . \vartheta_{p}$ is obtained from all $\vartheta_{p}^{(j)}$ according to function (3), and $\xi_{p}$ is calculated similar to $\vartheta_{p}$.

$$
\vartheta_{p}=\sum_{j=1}^{n} \alpha^{(j)} \cdot \vartheta_{p}^{(j)}
$$

Where $\alpha^{(j)}$ is the weight of $\vartheta_{p}^{(j)}, \sum_{j=1}^{n} \alpha^{(j)}=1$.In the process of determining weight $\alpha^{(j)}$, the correlation between state indicator $x$ and observation indicator $y^{(j)}$ should be considered. If the correlation between $x$ and $y^{(j)}$ is large, the weight $\alpha^{(j)}$ should be large; in contrast, the weight $\alpha^{(j)}$ is small. To address this, Kendall rank correlation method is proposed to be used to obtain weight $\alpha^{(j)}$. Set $\tau^{(j)}$ as the Kendall rank correlation coefficient of $x$ and $y^{(j)}$, and it can be known that $\tau^{(j)}$ is proportional to the correlation between $x$ and $y^{(j)}$ according to Kendall rank correlation definition [1]. The weight $\alpha^{(j)}$ can be calculated as

$$
\alpha^{(j)}=\frac{\tau^{(j)}}{\sum_{q=1}^{n} \tau^{(q)}}
$$

(d) Put $\theta_{p}=\left(\vartheta_{p}, \xi_{p}, \varphi_{p}^{(1)}, \varepsilon_{p}^{(1)}, \cdots, \varphi_{p}^{(n)}, \varepsilon_{p}^{(n)}\right)$ into $(p+1)$ th iteration of parameters estimation process if $p<L, L$ is the total number of iterations. $\vartheta_{L}$ and $\xi_{L}$ are the parameters estimation results that can be used for RUL prediction.

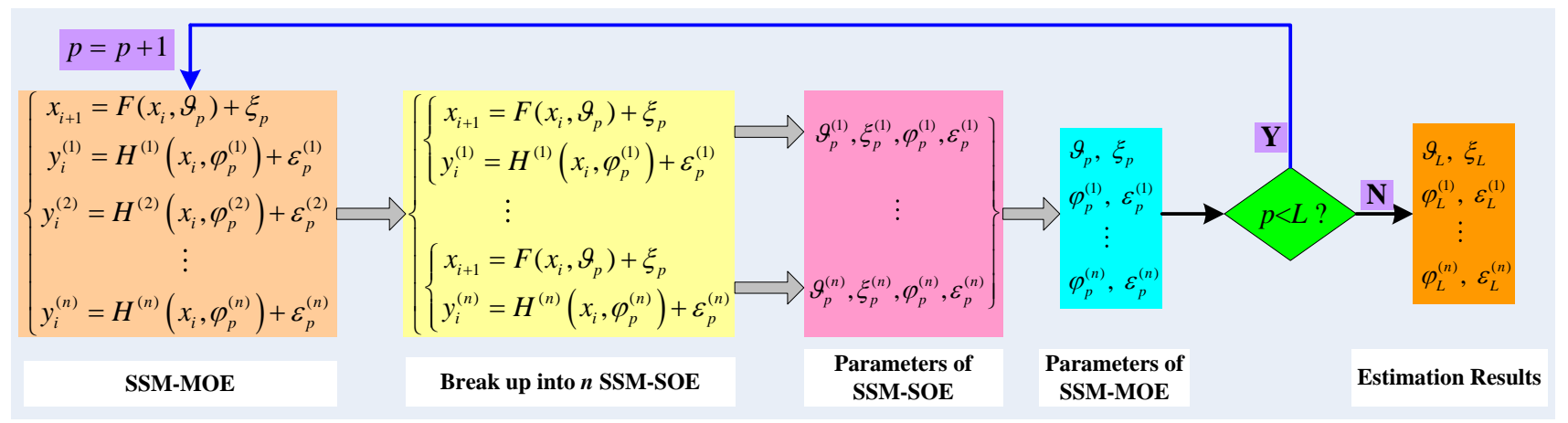

Figure 2. Parameters estimation process of SSM-MOE

\section{Simulation study}

\subsection{SSM-MOE based on Gamma process}

Simulation method is used to compare remaining useful life (RUL) prediction by traditional SSM-SOE and SSM-MOE. In order to simplify the calculation, the SSM-MOE is considered to have two observation equations in this simulation study.

The SSM-MOE is shown in Formula (5). Formula (5a) is the degradation state evolution function. The system degradation process is considered as smooth Gamma process [4,5], and the shape-parameter $\eta(\Delta t)$ is assumed to be $\eta(\Delta t)=a \cdot\left(t_{i}-t_{i-1}\right)$. Formula (5b) and Formula (5c) are the first and second observation equations, respectively. The relationship between observation indicator and state indicator is linear in the first observation equation, and it is nonlinear $(d \neq 1)$ in the second observation equation. 


$$
\left\{\begin{array}{l}
x_{i}-x_{i-1} \sim \operatorname{Gamma}\left(a \cdot\left(t_{i}-t_{i-1}\right), \xi\right) \\
y_{i}=b \cdot x_{i}+\varepsilon \\
w_{i}=c \cdot\left(x_{i}\right)^{d}+\delta
\end{array}\right.
$$

Where, observation noises $\varepsilon, \delta$ are assumed to be normal distribution, $\varepsilon \sim N\left(0, \sigma_{y}^{2}\right)$ and $\delta \sim N\left(0, \sigma_{w}^{2}\right)$.

There are three SSM that can be established in this study: Mod 1 is composed of formulas (5a) and (5b), Mod 2 is composed of formulas (5a) and (5c), Mod 3 is composed of formulas (5a), (5b) and (5c). It is easy to find, Mod 1 and Mod 2 are traditional SSM-SOE, Mod 3 is a SSM-MOE.

\subsection{Parameter estimation and RUL prediction}

As Mod 2 is more complex than Mod 1, take Mod 2 as an example to illustrate the parameters estimation method of SSMSOE in the simulation study. If monitoring time sequence $t_{0: m}$ and observation indicator sequence $w_{0: m}$ have been given, we can set up the complete $\log$-likelihood function of Mod 2, and then take the partial derivative of $a, \xi, c, d$ and $\sigma_{w}$, respectively $[8,9]$. As a result, the relationships between model parameters are obtained as follows:

$$
\begin{gathered}
\sum_{i=2}^{m}\left(\ln \xi+\frac{\Gamma^{\prime}\left(a \cdot\left(t_{i}-t_{i-1}\right)\right)}{\Gamma\left(a \cdot\left(t_{i}-t_{i-1}\right)\right)}-E\left(\ln \left(x_{i}-x_{i-1}\right)\right)\right) \cdot\left(t_{i} \cdot \ln t_{i}-t_{i-1} \cdot \ln t_{i-1}\right)=0 \\
\xi=\frac{E\left(x_{m}\right)-E\left(x_{1}\right)}{a \cdot\left(t_{m}-t_{1}\right)} \\
c=\frac{\sum_{i=1}^{m} w_{i} \cdot E\left(\left(x_{i}\right)^{d}\right)}{\sum_{i=1}^{m} E\left(\left(x_{i}\right)^{2 d}\right)} \\
\sum_{i=1}^{m} E\left(\left(x_{i}\right)^{2 d}\right) \cdot \sum_{i=1}^{m}\left(w_{i} \cdot E\left(\left(x_{i}\right)^{d} \ln x_{i}\right)\right)-\sum_{i=1}^{m}\left(w_{i} \cdot E\left(\left(x_{i}\right)^{d}\right)\right) \cdot \sum_{i=1}^{m} E\left(\left(x_{i}\right)^{2 d} \cdot \ln x_{i}\right)=0 \\
\sigma_{w}=\sqrt{\frac{1}{m} \cdot \sum_{i=1}^{m}\left(\left(w_{i}\right)^{2}-2 w_{i} \cdot c \cdot E\left(\left(x_{i}\right)^{d}\right)+c^{2} \cdot E\left(\left(x_{i}\right)^{2 d}\right)\right)}
\end{gathered}
$$

Where $E(Z)$ is the average of $Z$.

It is can be found from formulas (6) (10) that all parameters can be calculated as long as $E\left(x_{i}\right), E\left(\left(x_{i}\right)^{d}\right), E\left(\left(x_{i}\right)^{2 d}\right)$ and $E\left(\ln \left(x_{i}-x_{(i-1)}\right)\right)$ are known. In order to reduce the uncertainty of calculated results, $E\left(x_{i}\right), E\left(\left(x_{i}\right)^{d}\right), E\left(\left(x_{i}\right)^{2 d}\right)$ and $E\left(\ln \left(x_{i}-x_{(i-1)}\right)\right)$ can be estimated by particle filter (PF) [8,9] as: $E\left(x_{i}\right) \approx \sum_{s=1}^{N_{s}} k_{i}^{s} \cdot x_{i}^{s}, \quad E\left(\left(x_{i}\right)^{d}\right) \approx \sum_{s=1}^{N_{s}} k_{i}^{s} \cdot\left(x_{i}^{s}\right)^{d}, \quad E\left(\left(x_{i}\right)^{2 d}\right) \approx \sum_{s=1}^{N_{s}} k_{i}^{s} \cdot\left(x_{i}^{s}\right)^{2 d}$, $E\left(\ln \left(x_{i}-x_{i-1}\right)\right) \approx \sum_{s=1}^{N_{s}} k_{i}^{s} \cdot \ln \left(x_{i}^{s}-x_{i-1}^{s}\right)$, where $N_{s}$ is the particle number of $x_{i}, x_{i}^{s}$ is the sth particle of $x_{i}$, and $k_{i}^{s}$ is the weight of $x_{i}^{s}$.

Set the system degradation state quantity is $x_{c}$ at time $t_{c}$, and the failure thresholdis $X_{f}$, the cumulative distribution function (CDF) of RUL $t_{r}$ can be written as 


$$
F\left(t_{r} \mid w_{0: c}\right)=\sum_{i=1}^{N_{s}} \frac{\Gamma\left(a \cdot t_{r},\left(X_{f}-x_{c}^{s}\right) / \xi\right)}{\Gamma\left(a \cdot t_{r}\right)} \cdot k_{c}^{s}
$$

The system average RUL is

$$
T_{r}=\int_{0}^{\infty}\left(1-F\left(t_{r} \mid w_{0: c}\right)\right) d t
$$

\subsection{Degradation simulation}

$R U L_{A}$ is the actual RUL in simulation, and $R U L_{P}$ is the predicted RUL after model parameters estimation. The relative error of $R U L_{A}$ and $R U L_{P}$ is proposed as an evaluation criterion, and it can be written as

$$
r=\frac{\left|R U L_{A}-R U L_{P}\right|}{R U L_{A}} \times 100 \%
$$

The greater the relative error $r$, the greater the predicted RUL deviates from actual RUL.

In this simulation study, the parameters used to generate the simulated data are $\theta=\left(a, \xi, b, \sigma_{y}, c, d, \sigma_{w}\right)=(0.2,0.7,2,1,1,1.4,1)$. The system degradation failure threshold is $X_{f}=10$, and the exact system failure time is assumed to be observable. Degradation state indicator $x$ is inspected every 1 hour, and observation indicators $y$ and ware are monitored every 0.25 hour and 0.5 hour, respectively. A total of 200 sets of data are simulated. Meanwhile, there are 200 particles that are used during PF smoothing in parameters evaluation process, and weights of observation indicators $\alpha_{y}$ and $\alpha_{w}$ are calculated in each set of data. Four cases of RUL prediction are considered in every simulation, namely the actual RUL are nearly $20 \mathrm{~h}, 15 \mathrm{~h}, 10 \mathrm{~h}$ and $5 \mathrm{~h}$,

\begin{tabular}{|c|c|c|c|c|}
\hline Models & Actual RUL (h) & Mean relative error & Actual RUL (h) & Mean relative error \\
\hline Mod 1 & \multirow{3}{*}{ Nearly 20.0} & $8.23 \%$ & \multirow{3}{*}{ Nearly 10.0} & $7.05 \%$ \\
\hline Mod 2 & & $9.36 \%$ & & $7.84 \%$ \\
\hline Mod 3 & & $7.42 \%$ & & $5.71 \%$ \\
\hline Mod 1 & \multirow{3}{*}{ Nearly 15.0} & $7.61 \%$ & \multirow{3}{*}{ Nearly 5.0} & $6.08 \%$ \\
\hline Mod 2 & & $8.45 \%$ & & $6.52 \%$ \\
\hline Mod 3 & & $6.90 \%$ & & $4.56 \%$ \\
\hline
\end{tabular}
respectively. The mean relative errors of $R U L_{A}$ and $R U L_{P}$ are shown in Table 1.

Table 1. Mean relative errors of RUL evaluation results

As shown in Table 1, it can be found that: (a) For the same model, the larger the RUL, the greater the mean relative error. This is because the larger the RUL, the less historical data can be used for RUL prediction. (b) When the RUL is nearly the same, the mean relative error of Mod 2 is the largest, and it is the smallest in Mod 3. Due to the sampling interval of observation indicator $w$ is larger than $y$, the historical data for RUL prediction in Mod 2 is the least, and it is the most in Mod 3. It also means that the proposed SSM-MOE is more accurate than traditional SSM-SOE in system degradation prediction, because SSM-MOE uses more historical observation data to reduce uncertainty.

\section{Conclusions}

This paper puts forward a state-space model having multiple observation equations that can be applied to multiple observation indicators with different sampling time. Model formulation and parameter estimation method of proposed model are studied. A simulation study is conducted to show the advantage of SSM-MOE compared to traditional SSM-SOE in RUL estimation. The sampling times and observation equations are not the same for different observation indicators in the simulation study. The simulation results showed that SSM-MOE is more accurate than SSM-SOE in system degradation prediction.

In engineering practice, a system state indicator may be indirectly monitored through multiple observation indicators at the same time. Sampling times of these observation indicators are different or the relationships between state indicator and different observation indicators are not the same. In these cases, SSM-MOE is more suitable than SSM-SOE in system degradation prediction. 


\section{Acknowledgements}

This research was partially supported by the National Natural Science Foundation under grant number 71401173.

\section{References}

1. G. Kordelas and P. Daras, "Robust SIFT-based feature matching using Kendall's rank correlation measure," IEEE International Conference on Image Processing, pp. 325-328, 2009. (DOI: 10.1109/ICIP.2009.5413514)

2. Z. Li, Z. J. He, Y. Y. Zi, and X. F. Chen, "Bearing Condition Monitoring Based on Shock Pulse Method and Improved Redundant Lifting Scheme," Mathematics and Computers in Simulation, vol. 79, no. 3, pp. 318-338, 2008. (DOI: 10.1016/j.matcom.2007.12.004)

3. H. K. Li, H. Li, S.J. Liu, and M. Cong, "Reliability Estimation Based on Moving Average and State Space Model for Rolling Element Bearing," International Journal of Performability Engineering, vol. 11, no. 3, pp. 243-256, 2015.

4. X. L. Ni, J. M. Zhao, J. C. Chen, and H. P. Li, "Reliability Modeling for Two-stage Degraded System Based on Cumulative Damage Model," International Journal of Performability Engineering, vol. 12, no. 1, pp. 89-94, 2016.

5. J. M. Noortwijk, "A Survey of the Application of Gamma Processes in Maintenance," Reliability Engineering and System Safety, vol. 94, no. 1, pp.94:2-21, 2009. (DOI: 10.1016/j.ress.2007.03.019)

6. M. E. Orchard and G. J. Vachtsevanos, "A Particle-Filtering Approach for On-line Fault Diagnosis and Failure Prognosis," Transactions of the Institute of Measurement and Control, vol. 31, pp. 221-246, 2009.

7. J. Z. Sun, H. F. Zuo, W. B. Wang, and M. G. Pecht, "Application of a State Space Modeling Technique to System Prognostics Based on a Health Index for Condition-Based Maintenance," Mechanical Systems and Signal Processing, vol. 28, pp. 585-596, 2012. (DOI:10.1016/j.ymssp.2011.09.029)

8. Y. F. Zhou, L. Ma, J. Mathew, Y. Sun, and R. Wolff, "Asset Life Prediction Using Multiple Degradation Indicators and Failure Events: a Continuous State Space Model Approach," EKSPLOATACJA I NIEZAWODNOSC-Maintenance and Reliability, vol. 4, pp. 72-81, 2009.

9. Y. F. Zhou, Y. Sun, J. Mathew, R. Wolff, and L. Ma, "Latent Degradation Indicators Estimation and Prediction: A Monte Carlo Approach," Mechanical system and Signal Processing, vol. 25, no. 1, pp. 222-236, 2011. (DOI: 10.1016/j.ymssp.2010.08.012)

Xianglong Ni received the B.S. degree from Jimei University, Xiamen, China, in 2011. He received the M.S. and Ph.D. degree in Mechanical Engineering College, Shijiazhuang, China, in 2013 and 2017, respectively. His main research interests include gearbox fault prognosis, degradation modeling, and maintenance decision-making.

Xin Zhang received the B.S. degree from Dalian Maritime University, Dalian, China, in 2014, and he received the M.S. degree from Mechanical Engineering College, Shijiazhuang, China, in 2016. He is now a Ph.D. student studying in Mechanical Engineering College, Shijiazhuang, China. His main research interests include bearing fault prognosis and degradation modeling.

Jianmin Zhao received his Ph.D. degree from the University of Birmingham, Birmingham, UK. Currently, he is a Professor at Mechanical Engineering College, Shijiazhuang, China. He has authored and co-authored more than 60 technical journal articles and conference papers. He is now leading a group in Key Lab of Reliability Centered Maintenance for application of condition based maintenance technologies to vehicles, wind turbines and helicopters. His areas of interest include degradation modeling for mechanical components, maintenance decision-making, and Warranty return policy.

Haiping Li received the B.S. degree from Beijing Institute of Technology, Beijing, China, in 2012, and he received the M.S. degree from Mechanical Engineering College, Shijiazhuang, China, in 2014. He is now a Ph.D. student studying in Mechanical Engineering College, Shijiazhuang, China. His main research interests include planetary gearbox fault diagnosis and prognosis. 